PROCEEDINGS OF THE

AMERICAN MATHEMATICAL SOCIETY

Volume 135, Number 5, May 2007, Pages 1355-1363

S 0002-9939(06)08581-9

Article electronically published on October 27, 2006

\title{
LINEAR DIFFERENTIAL EQUATIONS IN THE UNIT DISC WITH ANALYTIC SOLUTIONS OF FINITE ORDER
}

\author{
RISTO KORHONEN AND JOUNI RÄTTYÄ
}

(Communicated by Juha M. Heinonen)

AbStract. A function $g$, analytic in the unit disc $D$, belongs to the weighted Hardy space $H_{q}^{\infty}$ if $\sup _{0 \leq r<1} M(r, g)\left(1-r^{2}\right)^{q}<\infty$, where $M(r, g)$ is the maximum modulus of $g(\bar{z})$ in the circle of radius $r$ centered at the origin. If $g$ belongs to $H_{q}^{\infty}$ for some $q \geq 0$, then it is said to be an $\mathcal{H}$-function. Heittokangas has shown that all solutions of the linear differential equation

$$
f^{(k)}+A_{k-1}(z) f^{(k-1)}+\cdots+A_{1}(z) f^{\prime}+A_{0}(z) f=0,
$$

where $A_{j}(z)$ is analytic in $D$ for all $j=0, \ldots, k-1$, are of finite order of growth in $D$ if and only if all coefficients $A_{j}(z)$ are $\mathcal{H}$-functions.

It is said that $g \in G_{p}$ when $p=\inf \left\{q \geq 0: g \in H_{q}^{\infty}\right\}$. In this study it is shown that if all coefficients $A_{j}(z)$ of $(\dagger)$ satisfy $A_{j} \in G_{p_{j}}$ for all $j=$ $0, \ldots, k-1$, then all nontrivial solutions $f$ of $(\dagger)$ satisfy

$$
\min _{j=1, \ldots, k} \frac{p_{0}-p_{j}}{j}-2 \leq \sigma_{M}(f) \leq \max \left\{0, \max _{j=0, \ldots, k-1} \frac{p_{j}}{k-j}-1\right\},
$$

where $p_{k}:=0$ and

$$
\sigma_{M}(f):=\limsup _{r \rightarrow 1^{-}} \frac{\log ^{+} \log ^{+} M(r, f)}{-\log (1-r)} .
$$

In addition, if $n \in\{0, \ldots, k-1\}$ is the smallest index for which

$$
\frac{p_{n}}{k-n}=\max _{j=0, \ldots, k-1} \frac{p_{j}}{k-j}
$$

then there are at least $k-n$ linearly independent solutions of $(\dagger)$ such that

$$
\sigma_{M}(f) \geq \max _{j=0, \ldots, k-1} \frac{p_{j}}{k-j}-2 .
$$

These results are a generalization of a recent result due to Chyzhykov, Gundersen and Heittokangas.

\section{INTRODUCTION AND STATEMENT OF RESULTS}

Wittich was the first to utilize Nevanlinna theory in the systematic study of growth properties of the solutions of the differential equation

$$
f^{(k)}+A_{k-1}(z) f^{(k-1)}+\cdots+A_{1}(z) f^{\prime}+A_{0}(z) f=0
$$

with entire coefficients. He showed that all solutions of (1.1) are entire functions of finite order if and only if all coefficients are polynomials [13. Later on, more

Received by the editors June 15, 2005 and, in revised form, November 22, 2005.

2000 Mathematics Subject Classification. Primary 34M10; Secondary 30D35.

The research reported in this paper was supported in part by the Academy of Finland grant numbers 204819 and 210245 and by the MEC Spain MTM2005-07347. 
detailed studies on the growth of solutions were done by different authors; see, for instance, 2, 5, 9, 11, 12. In particular, Gundersen, Steinbart and Wang listed all possible orders of growth of entire solutions of (1.1) in terms of the degrees of the polynomial coefficients [6].

Analogously to Wittich's classification of finite-order entire solutions of (1.1), Heittokangas showed that all solutions of (1.1) are finite-order analytic functions in the unit disc if and only if the coefficients are $\mathcal{H}$-functions [7]. A more precise study on the growth of the solutions was given by Chyzhykov, Gundersen and Heittokangas 4. They considered certain natural subclasses of the general linear equation (1.1) assuming that the coefficients have a particular preassigned growth within the class of $\mathcal{H}$-functions. The exact formulation is as follows. First, the weighted Hardy space $H_{q}^{\infty}$ consists of those functions $g$, analytic in the unit disc, such that

$$
\sup _{0 \leq r<1} M(r, g)\left(1-r^{2}\right)^{q}<\infty
$$

where $M(r, g)$ is the maximum modulus of $g(z)$ in the circle of radius $r$ centered at the origin.

If $g$ belongs to $H_{q}^{\infty}$ for some $q \geq 0$, then it is said to be an $\mathcal{H}$-function 11 In particular, it is said that $g \in G_{p}$ when $p=\inf \left\{q \geq 0: g \in H_{q}^{\infty}\right\}$. The following theorem, due to Chyzhykov, Gundersen and Heittokangas [4, gives a sharp upper bound for the order of analytic solutions of (1.1) when the coefficients $A_{1}(z), \ldots, A_{k-1}(z)$ vanish identically.

Theorem A. Let $f$ be a solution of

$$
f^{(k)}+A(z) f=0,
$$

where $k \in \mathbb{N}$ and $A \in G_{p}$ for some $p$ satisfying $p \geq k$. Then

$$
\sigma_{M}(f):=\limsup _{r \rightarrow 1^{-}} \frac{\log ^{+} \log ^{+} M(r, f)}{-\log (1-r)} \leq \frac{p}{k}-1,
$$

where $\log ^{+} x:=\max \{0, \log x\}$.

There are two different ways to define order of growth in the unit disc. In addition to $\sigma_{M}(f)$ defined in (1.3), the quantity

$$
\sigma(f):=\limsup _{r \rightarrow 1^{-}} \frac{\log ^{+} m(r, f)}{-\log (1-r)}
$$

where $m(r, f)$ is the Nevanlinna proximity function of $f$, is often used to describe the growth of an analytic function in the unit disc. Although in the complex plane analogously defined growth orders are equal for all entire functions, in the unit disc we only have the inequality

$$
\sigma(f) \leq \sigma_{M}(f) \leq \sigma(f)+1,
$$

which is the best possible in the sense that there are analytic functions $g$ and $w$ such that $\sigma_{M}(g)=\sigma(g)$ and $\sigma_{M}(w)=\sigma(w)+1$. The following theorem gives a lower bound for the order of growth of solutions of (1.1) when the growth of the coefficient $A_{0}(z)$ is in a sense dominant [4].

\footnotetext{
${ }^{1}$ The union of all $H_{q}^{\infty}$-spaces is also known as the Korenblum space $A^{-\infty}[10$.
} 
Theorem B. Let $f$ be a nontrivial solution of (1.1), where $A_{j} \in G_{p_{j}}$ for $j=$ $0,1, \ldots, k-1$. If

$$
p_{j} \leq \frac{k-j}{k} p_{0}
$$

for $j=1,2, \ldots, k-1$, then

$$
\frac{p_{0}}{k}-2 \leq \sigma(f) \leq \sigma_{M}(f)
$$

The purpose of this paper is to study the general linear differential equation (1.1) where the coefficients are $\mathcal{H}$-functions. Our findings are summarized in the following theorem which contains Theorems $\mathrm{A}$ and $\mathrm{B}$ as a special case and improves in part [3, Theorems 1 and 2]. The lower bound in (1.7) below is obtained by the proof of [4, Theorem 2.3].

Theorem 1. Let $f$ be a nontrivial solution of (1.1), where $A_{j} \in G_{p_{j}}$ for $j=$ $0,1, \ldots, k-1$. Then the order of growth $\sigma_{M}(f)$ satisfies

$$
\min _{j=1, \ldots, k} \frac{p_{0}-p_{j}}{j}-2 \leq \sigma(f) \leq \sigma_{M}(f) \leq \max \left\{0, \max _{j=0, \ldots, k-1} \frac{p_{j}}{k-j}-1\right\},
$$

where $p_{k}:=0$. Moreover, if $n \in\{0, \ldots, k-1\}$ is the smallest index for which

$$
\frac{p_{n}}{k-n}=\max _{j=0, \ldots, k-1} \frac{p_{j}}{k-j},
$$

then there are at least $k-n$ linearly independent solutions of (1.1) such that

$$
\max _{j=0, \ldots, k-1} \frac{p_{j}}{k-j}-2 \leq \sigma(f) \leq \sigma_{M}(f) .
$$

If the maximum in (1.9) is attained when $j=0$, then the left-hand sides of inequalities (1.7) and (1.9) are equal.

We discuss the sharpness of the assertions of Theorem 1 as follows. For $\beta \geq 1$ the functions $f_{1}(z)=\exp \left(\frac{\beta}{1-z}\right)^{\beta}$ and $f_{2}(z)=\exp \left(\frac{\beta}{1-z}\right)^{\beta+1}$ are linearly independent solutions of

$$
f^{\prime \prime}+A_{1}(z) f^{\prime}+A_{0}(z) f=0
$$

where

$$
A_{0}(z)=\frac{\beta^{\beta+1}(\beta+1)}{(1-z)^{\beta+2}(z+\beta)}+\frac{\beta^{2 \beta+2}(\beta+1)}{(1-z)^{2 \beta+3}}
$$

and

$$
A_{1}(z)=\frac{\beta^{\beta+1}(z-\beta-2)}{(1-z)^{\beta+2}}-\frac{(\beta+1)(z+\beta+1)}{(z+\beta)(1-z)} .
$$

Clearly $A_{0} \in G_{2 \beta+3}$ and $A_{1} \in G_{\beta+2}$, and

$$
\sigma\left(f_{1}\right)=\min _{j=1,2} \frac{p_{0}-p_{j}}{j}-2=\beta-1,
$$

which shows that the lower bound may be attained for $\sigma(f)$ in (1.7). On the other hand, $n=1$ is the smallest index such that (1.8) holds, and

$$
\max _{j=0,1} \frac{p_{j}}{k-j}-2=\beta=\sigma\left(f_{2}\right)>\sigma\left(f_{1}\right),
$$


which means that there is exactly one solution in the base $\left\{f_{1}, f_{2}\right\}$ such that (1.9) is satisfied. Therefore also the estimate for the number of solutions with fast growth is the best possible in this case. Finally, consider (1.10) with

$$
A_{0}(z)=4 \frac{\beta\left(\frac{1+z}{1-z}\right)^{\beta}+\beta+1}{(1-z)^{2}(1+z)^{2}}
$$

and

$$
A_{1}(z)=-2 \frac{\beta\left(\frac{1+z}{1-z}\right)^{\beta}+z+\beta+2}{(1-z)(1+z)} .
$$

Then $A_{1} \in G_{\beta+1}$ is the maximal coefficient in the sense of (1.8), $A_{0} \in G_{\beta+2}$, and (1.10) is solved by $f_{1}(z)=\frac{1+z}{1-z} \exp \left(\frac{1+z}{1-z}\right)^{\beta}$ and $f_{2}(z)=\frac{1+z}{1-z}$, which are linearly independent. Now

$$
\max _{j=0,1} \frac{p_{j}}{k-j}-1=\sigma_{M}\left(f_{1}\right)=\beta
$$

which demonstrates that also the upper bound in (1.7) may be attained.

\section{Proof of Theorem 1}

Since Wiman-Valiron theory, a powerful tool to study entire solutions of linear differential equations, does not have a sufficiently efficient analogue in the unit disc for our purposes, we resort to different methods in the proof of Theorem 1 The upper bound for the order of growth in (1.7) is obtained by a recent growth estimate for analytic solutions of linear differential equations [8]. The lower bound in (1.7) and the estimate for the number of fast-growing linearly independent solutions is obtained by combining the standard order-reduction procedure for linear differential equations [6, 11] together with logarithmic derivative estimates in the unit disc [4].

2.1. Preparation for the proof. The order-reduction procedure is summarized

in the following lemma, which is a modification of [6, Lemma 6.4] to be used in the unit disc.

Lemma C. Let $f_{0,1}, f_{0,2}, \ldots, f_{0, m}$ be $m \geq 2$ linearly independent meromorphic solutions of

$$
y^{(k)}+A_{0, k-1}(z) y^{(k-1)}+\cdots+A_{0,0}(z) y=0, \quad k \geq m,
$$

where $A_{0,0}, \ldots, A_{0, k-1}$ are meromorphic functions in the unit disc. For $1 \leq q \leq$ $m-1$, set

$$
f_{q, j}=\left(\frac{f_{q-1, j+1}}{f_{q-1,1}}\right)^{\prime}, \quad j=1,2, \ldots, m-q .
$$

Then $f_{q, 1}, f_{q, 2}, \ldots, f_{q, m-q}$ are linearly independent meromorphic solutions of

$$
y^{(k-q)}+A_{q, k-q-1}(z) y^{(k-q-1)}+\cdots+A_{q, 0}(z) y=0,
$$

where

$$
A_{q, j}(z)=\sum_{n=j+1}^{k-q+1}\left(\begin{array}{c}
n \\
j+1
\end{array}\right) A_{q-1, n}(z) \frac{f_{q-1,1}^{(n-j-1)}(z)}{f_{q-1,1}(z)}
$$

for $j=0,1, \ldots, k-q-1$. Here $A_{n, k-n} \equiv 1$ for all $n=0,1, \ldots, q$. 
When reducing the order of the linear equation (1.1) we end up with an equation which has in general meromorphic coefficients. We introduce some additional notation to deal with this issue. Given a set $F \subset[0,1)$ of infinite logarithmic measure $\int_{F} d t /(1-t)=\infty$, we say that the space $H_{q}^{\infty}(F)$ consists of all meromorphic functions $g$ in the unit disc such that

$$
\sup _{\substack{|z|<1 \\|z| \in F}}|g(z)|\left(1-|z|^{2}\right)^{q}<\infty .
$$

Moreover, it is said that $g \in G_{p}(F)$ when $p=\inf \left\{q \geq 0: g \in H_{q}^{\infty}(F)\right\}$.

Lemma 2. Let $F=[0,1) \backslash E$ where $E \subset[0,1)$ is a set of finite logarithmic measure $\int_{E} d t /(1-t)<\infty$, let $g \in G_{p}(F)$ for some $p>0$, and let $\varepsilon>0$. Then there exists a set $\widetilde{F}_{\varepsilon} \subset F$ of infinite logarithmic measure $\int_{\widetilde{F}_{\varepsilon}} d t /(1-t)=\infty$ such that

$$
\liminf _{\substack{r \rightarrow 1^{-} \\ r \in \bar{F}_{\varepsilon}}} \frac{\log ^{+} M(r, g)}{-\log (1-r)} \geq p-\varepsilon \text {. }
$$

Proof. Assume that

$$
M(r, g) \leq\left(\frac{1}{1-r}\right)^{p-\varepsilon}
$$

for all $r$ outside of a set of finite logarithmic measure. Then by [1, Lemma $\mathrm{C}$ ] there exists a constant $d \in(0,1)$ such that if $s(r)=1-d(1-r)$, then

$$
M(r, g) \leq\left(\frac{1}{1-s(r)}\right)^{p-\varepsilon}
$$

without an exceptional set. Since $1 /(1-s(r))=1 /(d(1-r))$, it follows by (2.2) that $g \in H_{p-\varepsilon}^{\infty}$, which contradicts the assumption $g \in G_{p}(F)$. We conclude that there is a set $\widetilde{F}_{\varepsilon}$ of infinite logarithmic measure such that

$$
M(r, g)>\left(\frac{1}{1-r}\right)^{p-\varepsilon}
$$

for all $r \in \widetilde{F}_{\varepsilon}$. Inequality (2.1) follows immediately by (2.3).

2.2. The upper bound in (1.7). First, by [8, Theorem 5.1],

$$
M(r, f) \leq C_{1} \exp \left(k \int_{0}^{r} \sum_{j=0}^{k-1} M\left(t, A_{j}\right)^{\frac{1}{k-j}} d t\right),
$$

where $C_{1}$ is a positive constant, not depending on $r$. Second, by the assumption $A_{j} \in G_{p_{j}}$, for all $j=0, \ldots, k-1$, and thus

$$
M\left(r, A_{j}\right) \leq\left(\frac{1}{1-r}\right)^{p_{j}+\varepsilon}
$$

for any $\varepsilon>0$ when $r$ is sufficiently large. The upper bound in (1.7) follows by combining (2.4) and (2.5) since $\varepsilon>0$ is arbitrary. 
2.3. The lower bound in (1.7). We follow the proof of [4, Theorem 2.3]. By [4, Inequality (7.3)], there is a set $E \subset[0,1)$ of finite logarithmic measure $\int_{E} d t /$ $(1-t)<\infty$, and a positive constant $C$ such that for any $z$ satisfying $|z| \notin E$ and for any $\varepsilon>0$, we have

$$
\left|A_{0}(z)\right| \leq \sum_{j=1}^{k}\left|A_{j}(z)\right| \frac{f^{(j)}(z)}{f(z)} \mid \leq \sum_{j=1}^{k} \frac{C}{(1-|z|)^{p_{j}+j(\sigma(f)+2+\varepsilon)}},
$$

where $A_{k}(z) \equiv 1$ and $p_{k}=0$. Moreover, by [4, Identity (7.4)], there is a set $F \subset[0,1)$ of infinite logarithmic measure such that

$$
\lim _{\substack{r \rightarrow 1^{-} \\ r \in F}} M\left(r, A_{0}\right)(1-r)^{p_{0}-\varepsilon}=\infty .
$$

By choosing a sequence $\left|z_{n}\right|=r_{n} \rightarrow 1^{-}$such that $r_{n} \in F \backslash E$ for all $n \in \mathbb{N}$, (1.5), (2.6) and (2.7) yield

$$
\min _{j=1, \ldots, k} \frac{p_{0}-p_{j}}{j}-2 \leq \sigma(f) \leq \sigma_{M}(f)
$$

by letting $\varepsilon \rightarrow 0$.

Inequality (2.8) remains valid even if, given a set $E$ of finite logarithmic measure, the coefficients $A_{j}(z)$ of (1.1) are assumed to be in the spaces $G_{p_{j}}([0,1) \backslash E)$, rather than in $G_{p_{j}}$. This fact is needed at the end of Section 2.4 below.

2.4. The number of linearly independent solutions. If $n=0$ is the smallest index such that (1.8) holds, then

$$
\frac{p_{0}-p_{j}}{j}=\frac{p_{0}}{j}-\frac{p_{j}}{k-j} \cdot \frac{k-j}{j} \geq \frac{p_{0}}{j}-\frac{p_{0}}{k} \cdot \frac{k-j}{j}=\frac{p_{0}}{k}
$$

for all $j=1, \ldots, k-1$, and so the minimum on the left side of (1.7) is attained when $j=k$. Thus, by (1.7),

$$
\sigma_{M}(f) \geq \sigma(f) \geq \frac{p_{0}}{k}-2
$$

for all nontrivial solutions $f$ of (1.1), and therefore there are $k$ linearly independent solutions such that (1.9) is valid.

If $n \geq 1$ we assume to the contrary of the assertion that equation (1.1) has $n+1$ linearly independent solutions $f_{0, q}$ such that

$$
\sigma\left(f_{0, q}\right)<\alpha:=\max _{j=0, \ldots, k-1} \frac{p_{j}}{k-j}-2
$$

for all $q=1, \ldots, n+1$. Assume that $\alpha$ is strictly positive since otherwise there is nothing to prove. Denote $A_{0, m}(z)=A_{m}(z)$ and $A_{0, k} \equiv 1$. By Lemma C,

$$
f_{1, q}=\left(\frac{f_{0, q+1}}{f_{0,1}}\right)^{\prime}, \quad q=1, \ldots, n,
$$

are linearly independent solutions of

$$
y^{(k-1)}+A_{1, k-2}(z) y^{(k-2)}+\cdots+A_{1,0}(z) y=0
$$

with

$$
A_{1, m}(z)=\sum_{i=m+1}^{k}\left(\begin{array}{c}
i \\
m+1
\end{array}\right) A_{0, i}(z) \frac{f_{0,1}^{(i-m-1)}(z)}{f_{0,1}(z)}
$$


for $m=0,1, \ldots, k-2$. Since $\sigma\left(f_{0, q}\right)<\alpha$ for all $q=1, \ldots, n+1$, also $\sigma\left(f_{1, q}\right)<\alpha$ for all $q=1, \ldots, n$ by (2.9), and so (2.10) has $n$ linearly independent solutions $f_{1, q}$ satisfying $\sigma\left(f_{1, q}\right)<\alpha$.

We will now show that the maximality of the coefficient $A_{0, n}(z)$ of (1.1) implies the maximality of $A_{1, n-1}(z)$ in (2.10), in the sense of (1.8). By (2.11), we have

$$
\left|A_{0, m}(z)\right|-\left|S_{m}(z)\right| \leq\left|A_{1, m-1}(z)\right| \leq\left|A_{0, m}(z)\right|+\left|S_{m}(z)\right|
$$

where

$$
S_{m}(z):=\sum_{i=m+1}^{k}\left(\begin{array}{c}
i \\
m
\end{array}\right) A_{0, i}(z) \frac{f_{0,1}^{(i-m)}(z)}{f_{0,1}(z)} .
$$

By defining $\delta:=\left(\alpha-\sigma\left(f_{0,1}\right)\right) / 2$ and applying [4, Corollary 3.2(a)] into equation (2.13), there is a positive constant $C$ such that

$$
\left|S_{m}(z)\right| \leq \sum_{i=m+1}^{k} \frac{C}{(1-|z|)^{p_{i}+(i-m)\left(\sigma\left(f_{0,1}\right)+2+\delta\right)}}
$$

for all $z$ such that $r=|z|$ is outside of an exceptional set $E \subset[0,1)$ of finite logarithmic measure. Since $\sigma\left(f_{0,1}\right)+\delta=\alpha-\delta$, the assumption (1.8) and inequality (2.14) yield

$$
\left|S_{m}(z)\right|=O\left(\frac{1}{1-|z|}\right)^{\frac{p_{n}(k-m)}{k-n}-\delta}
$$

for all $m=1, \ldots, k-1$, and therefore by the assumption (1.8) and (2.12) there is an $\varepsilon^{\prime}>0$ such that

$$
\left|A_{1, m-1}(z)\right|=o\left(\frac{1}{1-|z|}\right)^{\frac{p_{n}(k-m)}{k-n}-\varepsilon^{\prime}}
$$

for all $m=1, \ldots, n-1$, where asymptotic relations (2.15) and (2.16) hold outside of a possible exceptional set $E$ of finite logarithmic measure. Denoting $F:=[0,1) \backslash E$, the assumption (1.8), (2.12) and (2.15) imply

$$
\limsup _{\substack{r \rightarrow 1^{-} \\ r \in F}} \frac{\log ^{+} M\left(r, A_{1, m-1}\right)}{-\log (1-r)} \leq \frac{p_{n}(k-m)}{k-n}
$$

for all $m=n, \ldots, k-1$. Moreover, since $A_{0, n} \in G_{p_{n}}$ by the assumption, Lemma 2 yields

$$
\liminf _{\substack{r \rightarrow 1^{-} \\ r \in F_{\varepsilon}}} \frac{\log ^{+} M\left(r, A_{0, n}\right)}{-\log (1-r)} \geq p_{n}-\varepsilon
$$

where $\varepsilon>0$ is arbitrary and $\widetilde{F}_{\varepsilon} \subset[0,1)$ is a set of infinite logarithmic measure. Therefore, by inequalities (2.12) and (2.15), we obtain

$$
\liminf _{\substack{r \rightarrow 1^{-} \\ r \in \vec{F}_{\varepsilon} \backslash E}} \frac{\log ^{+} M\left(r, A_{1, n-1}\right)}{-\log (1-r)} \geq p_{n}-\varepsilon
$$

for all $\varepsilon>0$, and thus $A_{1, n-1} \in G_{p_{n}}(F)$ by (2.17). Furthermore, by (2.16), the coefficient $A_{1, m}$ belongs to $G_{p_{1, m}}(F)$ for some nonnegative constants $p_{1, m}$ satisfying

$$
\frac{p_{1, m}}{k-1-m} \leq \frac{p_{n}}{k-n}=\frac{p_{1, n-1}}{k-1-(n-1)}
$$


for all $m=0, \ldots, k-2$, the inequality being strict for all $m=0, \ldots, n-2$. We conclude that $n-1$ is the smallest index such that

$$
\frac{p_{1, n-1}}{k-1-(n-1)}=\max _{j=0, \ldots, k-2} \frac{p_{1, j}}{k-1-j}
$$

This completes the first order-reduction step. By repeating the order-reduction $n$ times we end up with the linear equation

$$
y^{(k-n)}+A_{n, k-n-1}(z) y^{(k-n-1)}+\cdots+A_{n, 0}(z) y=0,
$$

where $A_{n, m} \in G_{p_{n, m}}\left(F_{n}\right)$ such that the complement of the set $F_{n} \subset[0,1)$ is of finite logarithmic measure, $p_{n, 0}=p_{n}$ and

$$
\frac{p_{n, 0}}{k-n}=\max _{j=0, \ldots, k-n-1} \frac{p_{n, j}}{k-n-j} .
$$

Therefore, by (2.8) all nontrivial solutions of (2.19) have the order of growth $\alpha$ at least. Since each order-reduction step reduces the number of linearly independent solutions of order less than $\alpha$ by one, and by antithesis equation (1.1) has $n+1$ linearly independent solutions $f_{0, q}$ such that $\sigma\left(f_{0, q}\right)<\alpha$, equation (2.19) has at least one nontrivial meromorphic solution $f_{n, 1}$ such that $\sigma\left(f_{n, 1}\right)<\alpha$. This contradiction completes the proof.

\section{REFERENCES}

1. S. Bank, A general theorem concerning the growth of solutions of first order algebraic differential equations, Compositio Math. 25 (1972), 61-70. MR0318546 (47:7093)

2. S. Bank and I. Laine, On the growth of meromorphic solutions of linear and algebraic differential equations, Math. Scand. 40 (1977), no. 1, 119-126. MR0454109 (56:12360)

3. Z.-X. Chen and K. H. Shon, The growth of solutions of differential equations with coefficients of small growth in the disc, J. Math. Anal. Appl. 297 (2004), no. 1, 285-304. MR2080381 (2005g:34224)

4. I. Chyzhykov, G. G. Gundersen and J. Heittokangas, Linear differential equations and logarithmic derivative estimates, Proc. London Math. Soc. 86 (2003), no. 3, 735-754. MR.1974397 (2004b:34224)

5. G. G. Gundersen, Finite order solutions of second order linear differential equations, Trans. Amer. Math. Soc. 305 (1988), no. 1, 415-429. MR0920167 (88j:34013)

6. G. G. Gundersen, E. M. Steinbart and S. Wang, The possible orders of solutions of linear differential equations with polynomial coefficients, Trans. Amer. Math. Soc. 350 (1998), no. 3, 1225-1247. MR1451603 (98h:34006)

7. J. Heittokangas, On complex differential equations in the unit disc, Ann. Acad. Sci. Fenn. Math. Diss. No. 122 (2000), 54 pp. MR1763775 (2001h:30025)

8. J. Heittokangas, R. Korhonen and J. Rättyä, Growth estimates for solutions of linear complex differential equations, Ann. Acad. Sci. Fenn. Math. 29 (2004), no. 1, 233-246. MR.2041951 (2004m:34199)

9. S. Hellerstein, J. Miles and J. Rossi, On the growth of solutions of $f^{\prime \prime}+g f^{\prime}+h f=0$, Trans. Amer. Math. Soc. 324 (1991), no. 2, 693-706. MR1005080 (91h:30047)

10. B. Korenblum, An extension of the Nevanlinna theory, Acta Math. 135 (1975), no. 3-4, 187-219. MR0425124 (54:13081)

11. I. Laine, Nevanlinna Theory and Complex Differential Equations, Walter de Gruyter, Berlin, 1993. MR.1207139 (94d:34008) 
12. I. Laine and $\mathrm{P}$. Wu, Growth of solutions of second order linear differential equations, Proc. Amer. Math. Soc. 128 (2000), no. 9, 2693-2703. MR1664418 (2000m:34009)

13. H. Wittich, Zur Theorie linearer Differentialgleichungen im Komplexen, Ann. Acad. Sci. Fenn. Ser. A. I. No. 379 (1966), 19 pp. MR0197828(33:5989)

Department of Mathematics, University of Joensud, P.O. Box 111, Fi-80101 Joensuu, FinLAND

E-mail address: risto.korhonen@joensuu.fi

Department of Mathematics, University of Joensud, P.O. Box 111, Fi-80101 Joensuu, FINLAND

E-mail address: jouni.rattya@joensuu.fi 